\title{
Observatorio
}

\section{Redes sociales: ¿modelos organizativos o servicios digitales?}

\author{
Por Juan Freire
}

\begin{abstract}
Resumen: Somos seres sociales y formamos redes desde nuestros inicios evolutivos. La red constituye un nuevo paradigma social y económico que ha sido denominado "Sociedad Red". Las personas que usan intensivamente las herramientas digitales fragmentan su identidad y la integran en múltiples redes locales y globales. Los servicios de redes sociales constituyen una simplificación y restricción de la diversidad de interacciones sociales. La combinación "a medida" de herramientas de la web 2.0 parece más interesante que el modelo Facebook.

Palabras clave: Redes sociales, Análisis, Evolución, Comportamiento, Organización social, Web 2.0, Comunicación.

Title: Social networks: organizational models or digital services?

Abstract: We are social beings and we have been creating networks since our inception. The network represents a new paradigm for social and economic development that has been called the "Network Society". People who use digital tools intensively are fragmenting their identity into multiple local and global net-

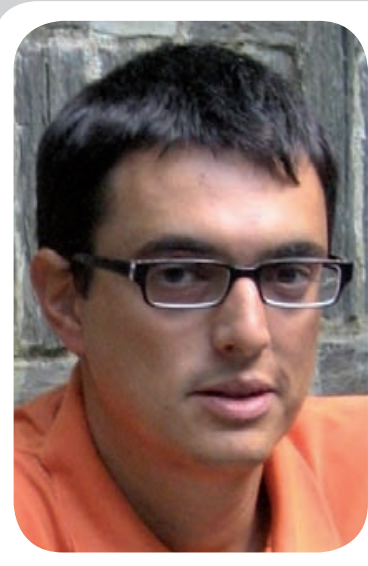

Juan Freire es doctor en Biología y profesor titular de la Universidad de A Coruña en la que fue Decano de la Facultad de Ciencias. Dirige el grupo de investigación en Recursos Marinos y Pesquerías que desarrolla numerosos proyectos de I+D. Cuenta con casi un centenar de publicaciones en revistas científicas internacionales y participa asiduamente en conferencias y grupos de trabajo relacionados con esta temática. Colabora como consultor con empresas, administraciones públicas y ONGs en sostenibilidad y gestión ambiental. Realiza proyectos y consultoría sobre innovación y estrategia en redes y organizaciones, en especial explorando el papel de la tecnología y los modelos de código abierto en las redes sociales, las universidades, las empresas y las ciudades. Colabora con los medios digitales ADN.es y Soitu.es. works. The social network services are a simplification and a restriction of the variety of social interactions. The combination of various Web 2.0 tools seems more interesting than the Facebook model.
\end{abstract}

Keywords: Social networks, Analysis, Evolution, Behaviour, Social organization, Web 2.0, Communication.

Freire, Juan. “Redes sociales: ¿modelos organizativos o servicios digitales?”. En: El profesional de la información, 2008, v. 17 , n. 6 , noviembre-diciembre, pp. 585-588.

DOI: 10.3145/epi.2008.nov.01

\section{Las redes como modelo social básico}

LAS REDES FORMAN PARTE de nuestra propia naturaleza biológica y cultural. Somos seres sociales y formamos redes desde nuestros inicios evolutivos. A lo largo de la evolución de nuestra especie y del desarrollo histórico de nuestras sociedades, la estructura de estas redes ha ido cambiando.

Así, inicialmente adoptaban en su mayoría un modelo organizativo extremo, conformando comunidades cerradas con escasa interacción con individuos externos. Utilizando la terminología actual, dominaban los vínculos fuertes. La cohesión, mediada por la identidad común dentro de la comunidad, era la mejor estrategia de supervivencia. En este sentido, las tribus y clanes, constituidos principalmente por vínculos familiares, fueron el modelo inicial y extremo de organización comunitaria cerrada. Este mismo modelo persiste hoy en día en muchas especies de primates, con las que compartimos gran parte de nuestra historia evolutiva. Pero además, estas formas sociales perviven hasta nuestros días, o renacen, en ciertos grupos humanos en que los "vínculos familiares" se han transformado en vínculos sociales y culturales relacionados con el nacionalismo o los movimientos de fans ${ }^{1}$.

A lo largo de la historia de la humanidad, las organizaciones tribales han dado paso a los sistemas basados en mercados y finalmente a las organizaciones en red. En la sociedad contemporánea, gracias a la tecnología de la información y las comunicaciones, las organizaciones sociales adquieren nuevas tipologías (más abiertas y con mayor frecuencia de vínculos débiles) y escalas (tamaños de grupo mayores) abriendo posibilidades insospechadas de colaboración y producción colectiva. De este modo las redes sociales pueden funcionar, y 
desarrollar proyectos para crear conocimiento, más allá de límites organizativos o institucionales. De hecho, la red constituye un nuevo paradigma social y económico en que nos hemos instalado en las últimas décadas del siglo XX y que ha sido denominado "Sociedad Red" En las fases previas, que se han denominado Sociedad de la Información o del Conocimiento, el valor estaba en la producción de conocimiento, mientras que en la Sociedad Red el conocimiento es ya extremadamente abundante y el verdadero valor está en la capacidad de gestionar el conocimiento: identificar la información relevante, integrándola y "remezclándola" para aplicarla en objetivos específicos. De este modo surge el papel de "comisario digital", en analogía al comisario o curator en el mundo del arte, como actor clave de la Sociedad $\mathrm{Red}^{3}$. Por otra parte, el propio desarrollo de las redes de comunicación y de información digitales ha contribuido a que vivamos en un contexto cada vez más complejo y con mayores incertidumbres en el que resulta esencial una organización en red, como estructura flexible y capaz de adaptarse rápidamente a cambios.

\section{"La red constituye un nuevo paradigma social y económico que ha sido denominado Sociedad Red"}

El Premio Nobel Ronald Coase propuso en 1937 la theory of the firm (en su artículo sobre la "naturaleza de la empresa") 4 para explicar a partir de los costes de transacción la existencia de las grandes empresas, un modelo organizativo que parecía innecesario en un sistema de mercado perfecto. Curiosamente, Ronald Coase fue en cierta medida el primero en predecir los cambios provocados por internet, dado que éstos se asocian en parte a la reducción de los costes derivados de la colaboración fuera de los límites de una empresa ${ }^{5}$. Dicho de otra forma, la razón de ser de una empresa es que es menos costoso organizarse bajo un paraguas organizativo que utilizando un sistema de mercado en el que tienes que negociar con proveedores externos todas las partes del proceso que quieres desarrollar. En esta idea se ha basado el paradigma analógico en la que han prosperado organizaciones concebidas como estructuras cerradas.

Pero la reducción de los costes de transacción externos, especialmente a partir de la década de 1990, es lo que explica la transformación de las organizaciones por el uso intensivo de las tecnologías de la información y las comunicaciones. Un caso extremo serían los fenómenos de innovación abierta, crowdsourcing ${ }^{6}$ o la capacidad de desarrollo de proyectos extremadamente complejos, como aquellos relacionados con el software libre o la propia Wikipedia, sin una estructura organi- zativa formal. La naturaleza propia de las empresas no es ya tanto producir y comercializar internamente como el organizar la producción y comercialización mediante cadenas o redes de colaboradores externos. La aparición de organizaciones informales ("organizarse sin organizaciones") en internet se explicaría a partir de la reducción de los costes (económicos, tiempo) de transacción que permite un incremento de los flujos e interacciones sociales ${ }^{7}$. Para obtener una visión completa que nos permita comprender los efectos transformadores de las tecnologías digitales, debemos tener en cuenta los efectos paralelos que provocan sobre las identidades individuales que afectan por tanto a las identidades colectivas (de las propias comunidades y redes). Las personas que usan intensivamente las herramientas digitales en sus prácticas y culturas fragmentan su identidad y la integran en múltiples redes locales y globales, al contrario que el modelo comunitario o las organizaciones formales y jerárquicas caracterizados por identidades unidimensionales.

No existen demasiadas estimaciones empíricas o teóricas del tamaño máximo de grupos en organizaciones humanas (cohesionados por vínculos fuertes). Quizás la mejor guía sea la que proporciona el antropólogo Robin Dunbar, conocida como número de Dunbar ${ }^{8}$, que estimó que los grupos de chimpancés podrían alcanzar un tamaño máximo de 55 individuos basándose en observaciones y en el estudio de los mecanismos de comunicación (la inversión de tiempo en este comportamiento sería lo que marcaría el límite superior). Según Dunbar, los humanos al contar con mayor capacidad cerebral podrían alcanzar tamaños de grupo de hasta 150 individuos. Se ha propuesto que la comunicación electrónica puede permitir ampliar este tamaño máximo, en especial con personas a las que nos unen conexiones débiles (muy comunes en las redes sociales digitales). Por supuesto, las nuevas redes soportadas en parte por la tecnología dependen en gran medida de los vínculos débiles y eso explicaría que alcancen muchas veces tamaños muy superiores y sigan siendo operativas.

\section{Redes sociales vs. servicios de redes sociales}

La reciente popularización de los servicios de redes sociales (que podríamos definir como instrumentos tecnológicos para desarrollar redes sociales), en su mayor parte en la forma de empresas que operan en internet, ha provocado una cierta confusión con el concepto de red social entendida como estructura organizativa y modelo social. Las entradas de la Wikipedia para ambos conceptos ponen de manifiesto estas diferencias 9 . Además, las iniciativas y servicios que se han denominado en conjunto como web 2.0 incluyen siempre utilidades de interacción social y por tanto todas ellas deberían 
considerarse medios sociales (en el sentido de que son instrumentos para la comunicación y la interacción y colaboración) o "sitios de redes sociales". Sin embargo sólo algunas de estas tecnologías se identifican como "redes sociales", y esta denominación se ha convertido en una forma de marca comercial.

Los sitios de internet auto-denominados "redes sociales" llevan ya años entre nosotros. LinkedIn o Xing son ya viejos conocidos dedicados al networking profesional con un éxito limitado. Pero en los últimos meses otros tipos de "redes", como MySpace y especialmente Facebook, han alcanzado un enorme éxito. De pronto, un modelo organizativo, la red, que sólo preocupaba a unos pocos se empieza a confundir con un tipo de herramientas, plataformas tecnológicas que aseguran permitir la creación de redes, que se hacen inmensamente populares en internet.

\section{"Los servicios de redes sociales aún simplifican y restringen la diversidad de interacciones sociales"}

Los servicios de redes sociales constituyen una simplificación y restricción de la diversidad de interacciones sociales. La tecnología no ha logrado, al menos hasta el momento, traducir completamente a código la compleja y diversa "gramática" de las relaciones entre seres humanos. Las relaciones humanas, incluyendo las digitales, son mucho más complejas y diversas de lo que estos servicios permiten. Por el contrario la web 2.0 cuenta con multitud de herramientas alternativas que permiten en gran medida obtener el mismo tipo de servicios que proporcionan las utilidades de redes sociales, pero además las decisiones de los usuarios seleccionando canales, sistemas y modelos de integración es lo que puede permitir que una red digital se aproxime a la riqueza y diversidad de una red social "completa". Las aplicaciones de la web 2.0 están diseñadas, de un modo finalista, para la interacción social. Cada una de ellas nos permite desarrollar sólo una pequeña parte de nuestro repertorio comportamental, pero el uso de múltiples canales por un mismo usuario, de un modo flexible y altamente personalizable, es lo que puede generar vínculos sociales más intensos e interesantes. Además, la distribución de las interacciones de una persona en diferentes sitios y aplicaciones (parte de la fragmentación de identidades que se citaba más arriba) constituye una forma de protección contra posibles intentos de control centralizado.

Todo lo anterior no quiere decir que estos servicios no puedan ser de gran utilidad para usos específicos, por ejemplo organizaciones que necesiten gestionar conjuntamente contenidos y usuarios en un ambiente controlado, como sucede en las empresas o las universidades. Pero al tiempo éste es precisamente uno de sus peligros: la capacidad para controlar las relaciones sociales digitales acabando con la apertura y libertad que define a internet, y resulta especialmente preocupante que se pueda asumir que las redes sociales desarrollan todo su potencial utilizando de modo exclusivo las tecnologías de estos servicios.

\section{“La combinación 'a medida' de herramientas de la web $\mathbf{2 . 0}$ parece más interesante que el modelo Facebook"}

El especialista en computación ubicua Adam Greenfield ha realizado una profunda crítica de los servicios de redes sociales, que denomina "antisocial networking" 10 , y proporciona diferentes razones para evitar el uso de este tipo de arquitecturas técnicas diseñadas para mediar las relaciones entre personas. Pero al tiempo reconoce las ventajas de algunas de estas plataformas que son abiertas y por tanto permiten el desarrollo de aplicaciones por terceros. De este modo es posible la portabilidad de perfiles y relaciones y se aumenta el número de servicios y utilidades ofrecidos. Pero, aun así, estas herramientas, tal como planteábamos antes, son absolutamente restrictivas dado que reducen la enorme diversidad de posibles relaciones entre personas que existen en el mundo real y en "el resto" de internet a unas pocas, y muy simples, opciones.

Podríamos identificar al menos tres tipos de simplificación de la realidad. En primer lugar, los sitios sociales me obligan a intercambiar las mismas informaciones con todos mis contactos calificados a un mismo nivel (por ejemplo, "amigos próximos"), algo que no sucede en el mundo real. Además, la complejidad y diversidad psicológica de nuestros sentimientos y relaciones con nuestros contactos dista mucho de poder ser representada con un mínimo de realismo con las escasísimas opciones que nos ofrecen los sistemas de internet. Por último, los procedimientos diseñados para el establecimiento de contactos en una red social son demasiado explícitos y obligan a los usuarios a ser poco sutiles en sus intenciones. Por ejemplo, nos obligan a rechazar a un "amigo" no deseado, o a tener que dar de baja una relación tras comprobar que no era de interés o conflictiva. La vida real permite tomar estas mismas decisiones sin tener que comunicarlas a un sistema.

Todas estas razones, además de su potencial capacidad para el control absoluto de las redes, hacen que las herramientas de redes sociales sean, como propone Adam Greenfield, una mala idea. Asumir lo que po- 
dríamos denominar un "modelo Facebook" como red social y espacio colaborativo limita considerablemente las opciones de creación e interacción, por lo que parecen más interesantes alternativas basados en modelos distribuidos de tecnologías para redes sociales (combinación "a medida" de herramientas de la web 2.0) que, por supuesto, requieren un nivel elevado de competencias digitales para poder gestionarlas e integrarlas ${ }^{11}$.

Pero además el desarrollo de redes sociales abiertas sobre plataformas privadas, como sucede en la mayor parte de casos en que se utilizan programas de la web 2.0, sean estas servicios de redes sociales o una combinación de otras aplicaciones, da lugar a un sistema que podría ser esencialmente inestable. Las redes sociales en internet funcionan bajo un contrato social subyacente en el que los usuarios admiten ciertos usos comerciales de su información y contenidos mientras no sobrepasen el límite en que superan a los beneficios por la disponibilidad de la plataforma ${ }^{12}$. La estabilidad a largo plazo de un modelo de este tipo es una cuestión que aún deberemos dilucidar en un futuro próximo.

\section{Referencias}

1. Ronfeldt, David. In search of how societies work. The tribes - the first and forever form, 2008. RAND working papers WR-433-RPC, $102 \mathrm{pp}$.

http://www.rand.org/pubs/working_papers/WR433/
2. Castells, Manuel. La era de la información: economía, sociedad y cultura, 2008, vol. I: La sociedad red ( $3^{\text {a }}$ edición). Alianza.

3. Freire, Juan (2008). "Conocimiento y usuarios en la cultura digital". Revista FRC, n. 16, pp. 53-57.

http://www.fcampalans.org/archivos/revista/13.jfreire.pdf

4. http://en.wikipedia.org/wiki/Theory_of_the_firm

http://www.cerna.ensmp.fr/Enseignement/CoursEcoIndus/SupportsdeCours/ COASE.pdf

5. Tapscott, Donald; Williams, Anthony D. Wikinomics: la nueva economía de las multitudes inteligentes, 2007. Paidos Ibérica.

6. Howe, Jeff. Crowdsourcing: how the power of the crowd is driving the future of business, 2008, Allen Lane. Random House Books.

7. Shirky, Clay. Here comes everybody: the power of organizing without organizations, 2008.

8. http://es.wikipedia.org/wiki/N\%C3\%BAmero_de_Dunbar

9. http://en.wikipedia.org/wiki/Social_network

http://en.wikipedia.org/wiki/Social_network_services

10. http://speedbird.wordpress.com/2007/12/09/antisocial-networking/

11. http://www.soitu.es/soitu/2007/12/26/pieldigital/1198709458_424459. html

12. Bauwens, Michel. La red social y sus contratos sociales, 2008, Minerva 09.

http://www.circulobellasartes.com/ag_ediciones-minerva-LeerMinervaCompleto.php?art=276

Juan Freire, Universidad de A Coruña

http://juanfreire.net/

\section{Congreso ISKO} ESPAÑA

Universidad Politécnica de Valencia

Valencia 11 - 13 Marzo 2009

\section{Nuevas perspectivas para la difusión y organización del conocimiento}

El Capítulo Español de ISKO celebrará su IX Congreso en Valencia durante los días 11, 12 y 13 de Marzo de 2009.

El evento será organizado por la Universidad Politécnica de Valencia, concretamente por el departamento de Comunicación Audiovisual, Documentación e Historia del Arte.

Bajo el lema "Nuevas perspectivas para la difusión y organización del conocimiento" se abordarán temas como: la epistemología del conocimiento, las redes sociales y el conocimiento colaborativo y la representación del conocimiento: modelado cuantitativo.

El congreso reunirá a un numeroso elenco de profesionales que trabajan en este ámbito y que ofrecerán diferentes perspectivas para avanzar en la nueva sociedad del conocimiento.
Por la cercanía de las Fallas la organización preparará una completa agenda de eventos para que los asistentes puedan conocer y disfrutar de estas fiestas.

\section{BLOQUES TEMÁTICOS}

Epistemología del conocimiento

Representación del conocimiento: modelado cuantitativo Las redes sociales y el conocimiento colaborativo

\section{INSCRÍBETE YA!}

\section{www.iskoix.org}

Camino de Vera $s / n_{\text {, }}$

46022 Valencia

tel. 963877000 (ext. 88924) 